\title{
Electrochemical Measurement of Antibody-Antigen Recognition Biophysics: Thermodynamics and Kinetics of Human Chorionic Gonadotropin (hCG) Binding to Redox-Tagged Antibodies
}

\author{
Lee I. Partington, ${ }^{1}$ Stephen L. Atkin, ${ }^{1,2,3}$ Eric S. Kilpatrick, ${ }^{2,4}$ \\ Samuel H. Morris, ${ }^{5}$ Mark Piper, ${ }^{5}$ Nathan S. Lawrence, ${ }^{6}$ Jay D. Wadhawan ${ }^{* 5,6}$ \\ ${ }^{1}$ Hull-York Medical School, The University of Hull, \\ Cottingham Road, Kingston-upon-Hull HU6 7RX, United Kingdom. \\ ${ }^{2}$ Department of Clinical Biochemistry, Hull Royal Infirmary, \\ Anlaby Road, Kingston-upon-Hull HU3 2JZ, United Kingdom. \\ ${ }^{3}$ Weill Cornell Medical College Qatar, Education City, \\ P. O. Box 24144, Doha, Qatar. \\ ${ }^{4}$ Department of Clinical Chemistry, Sidra Medical and Research Centre, \\ P. O. Box 26999, Doha, Qatar. \\ ${ }^{5}$ Department of Chemistry, The University of Hull, \\ Cottingham Road, Kingston-upon-Hull HU6 7RX, United Kingdom. \\ ${ }^{6}$ School of Engineering, The University of Hull, \\ Cottingham Road, Kingston-upon-Hull HU6 7RX, United Kingdom.
}

To be submitted to J. Electroanal. Chem.

${ }^{*}$ Author to whom all correspondence should be addressed.

E.mail: j.wadhawan@hull.ac.uk 


\section{Abstract}

The thermodynamics and kinetics of antigen binding under diffusive conditions to an electrode surface modified with ferrocene-tagged antibodies is studied within this work, and realised experimentally for the case of human chorionic gonadotropin (hGC) as the antigen with monoclonal anti-hCG antibodies immobilised on an electrode surface via a molecular wire platform. The formation of the antigen-antibody complex is monitored through the blocking of the ferrocene voltammetry, thereby enabling the fractional coverage of the electrode binding sites to be unravelled as a function of time. It is found that, at low antigen concentrations, a Frumkin adsorption isotherm fits the data, with repulsive interactions between bound antigens playing a significant rôle, with an affinity constant that is two orders of magnitude larger than in the case of an untagged antibody, suggesting that the chemical hydrophobicity of the redox tag may encourage stronger binding. Comparison of the experimental temporal data with relevant diffusion-adsorption models under activation control allows for the extraction of the kinetic parameters; at zero coverage, the rate constants for adsorption and desorption are, respectively, larger and smaller than the untagged antibody. The kinetic study enables the confirmation that this type of platform may be utilised for rapid $(15 \mathrm{~min})$ and quantitative electroimmunoassay. This is validated through proof-of-concept analytical measurements, yielding a limit of detection around $25 \mathrm{mIU} \mathrm{mL}^{-1}$ (corresponding to $2.7 \mathrm{ng} \mathrm{mL}^{-1}$ ) - a value used clinically for urine hCG measurements corresponding to around four weeks of gestational age.

Key words: Electroimmunoassay, Frumkin isotherm, partially blocked electrodes, molecular wire, chemoreception biophysics. 


\section{Introduction}

The hallmark of the specific adaptive immune response is the recognition of antigens (often proteins or carbohydrates) through binding to immunoglobulins (antibodies) present on the surface of $B$ lymphocytes ( $B$ cells) [1,2]. Such protein-protein binding, mediated by a mixture of electrostatic, hydrophobic, hydrogen bonding or van der Waals' forces, occurs with a strong affinity constant $\left(10^{8 \pm 3} \mathrm{M}^{-1}\right)$, and, at least for the case of monoclonal antibodies, is highly specific, so that the binding may discriminate between closely related compounds $[3,4]$.

The formation of such antibody-antigen complexes has been exploited extensively for the in vitro bioassay of a huge diversity of chemical structures and organisms [1-4], which has, inter alia, enabled a growth in analytical pointof-care sensors detecting antigenic disease biomarkers [5]. In general [6], two classes of measurement are used by such sensors; in a first approach, the binding assay uses an excess of the binding protein (antibody) that is conjugated with a suitable chemical tag, affording a stoichiometric assay. Such systems require separation of labelled and unlabelled antibody, typically through the use of a solid support. In a second approach, the binding protein is kept in limited amount, so that only a proportion of the antigen forms the antibody-antigen complex at equilibrium. In this scenario, initially bound, labelled, antigen may be displaced from the binding equilibrium by free, unlabelled antigen, enabling detection through the substoichiometric dilution principle [7].

Immunoassay using electrochemical methods is highly popular, owing to the ease through which developed sensing chemistry may be adapted to afford portable, low cost and reliable "dipstick" sensors of high sensitivity [8], especially for the case where the electrode surface itself is modified with antibody; rapid quantitative assay then relies on the antigen concentration in solution, the rate of antigen transport to the electrode surface and the kinetics of the interaction. In a previous work [9], we employed ferrocene-tagged antibodies attached to an electrode surface through a molecular wire platform to monitor the presence of biomarkers for cardiovascular disease in a physiological Krebs-Henseleit solution, where we demonstrated successful quantitative 
analysis using an antigen-incubation time of $20 \mathrm{~min}$. In this work, we are interested in unravelling the physicochemical whys and wherefores underpinning the chemoreception of that platform, to determine how low an antigen incubation time is possible, whilst reliably providing quantitative analysis. We use human chorionic gonadotropin (hCG) as the antigen, as the clinical interest is of increasing hCG concentration [6], so that we may work with known minimum biomarker levels (vide infra). This hormone is a $37 \mathrm{kDa}$ glycoprotein secreted by specialist syncytiotrophoblast cells of the developing placenta following implantation of a corpus leteum $[10,11]$. The presence of hCG can be detected in serum and urine, in some cases as early as three to four days following implantation, and therefore plays an important rôle in early pregnancy diagnosis [12]. However, the presence of hCG may indicate other health issues, including ectopic pregnancy [13], choriocarcinoma and hydatidiform moles [1416], thereby offering further clinical utility. The hormone comprises two dissimilar, non-covalently bound structures: the $\alpha$ and $\beta$ subunits [11]. The $\alpha-$ subunit is identical to those in the glycoprotein family, such as luteinizing hormone, whereas the $\beta$-subunit is unique to hCG and determines its immunological properties, providing an ideal target for specific detection via immunoassay techniques [10].

The majority of hCG immunoassays most commonly use enzyme-linked immunosorbent assay (ELISA) and other sandwich assay techniques [17], with recent advancements reported using chemiluminescence [18,19], fluorescence [20], and electrochemical immunosensor approaches [21-23], in addition to ondisc advancements [24]. The diagnostic cut-off for a positive reading is generally 25 milliinternational units per millilitre $\left(\mathrm{mIU} \mathrm{mL}^{-1}\right)$, corresponding to $2.7 \mathrm{ng} \mathrm{mL}^{-}$ 1 , although this varies significantly from test to test [25]. An hCG level of less than $5 \mathrm{mIU} \mathrm{mL} \mathrm{m}^{-1}$ generally indicates a negative result [26].

Previous work [27] has illustrated that for electrodes modified with anti-hCG immunoglobulins, the antibody orientation, in terms of binding site position, is extremely important for maximising the sensitivity of an hCG sensor. Moreover, neutron reflection studies have illustrated that for the ionic strengths employed 
in this work (typically $200 \mathrm{mM}$ ), hCG binding to IgG1 mouse monoclonal anti- $\beta$ hCG antibodies immobilised on silicon oxide wafers, is influenced by both steric hindrance and electrostatic interaction between the antigen and the wafer surface [28], and that decreasing the packing density of adsorbed antibody increases the antigen binding capacity [29]. It is clear that such steric crowding and steric hindrance effects on binding arising from the adsorbed antibody may be complemented by effects hitherto unreported pertaining to the mutual repulsion that results from the binding of the antigen to the antibody. In this paper, we examine the thesis that such antigen-antigen repulsions on binding to an antibody-modified surface may be manifested through alterations in the binding isotherm, which may be measured electrochemically, and which may additionally affect the rate constants for hCG binding, and thence the capability of this system in responding reliably for rapid immunoassay. 


\section{Theory}

\section{Development of a Global Antigen Coverage Parameter}

Our previous work on the development of a versatile platform for electroimmunoassay [9], was exploited as the foundation for the method described herein. In short, a redox-labelled monoclonal antibody was attached to an electrode surface through a covalently bound molecular wire, as schematically illustrated in Figure 1. The use of electrode-grafting by reducing diazonium functionalities means that a multilayer molecular wire platform is present [30]. In this work, we use the electrochemical reduction of a nitrobenzene diazaonium salt. Accordingly, in order to form an amide link with the antibody, the nitro-moieties need to be completely reduced to the amine. Owing to the complexity of this reduction [31], there is no easy way in which to coulomb count this; we therefore assume the resulting surface is sprinkled with nitro-, nitroso-, hydroxylamine and amine functionalities. This gives rise to a platform that is chemically non-uniform, and with a unique nanostructure. The antibody is likewise randomly modified with the redox tag - typically this is achieved through the formation of an amide linkage between the free primary amine moieties on the antibody and an aldehyde or acid functional group on the redox compound (such as ferrocenecarboxaldehyde or cisbis(isothiocyanato)bis(2,2'-bipyridyl-4,4'-dicarboxylato)ruthenium(II)). Whilst there is an abundance of free primary amines at the paratopes of the antibody, there is also the possibility of immobilising the redox functionality all over the antibody. Furthermore, as indicated in Figure 1, other than a statistical likelihood of the free carboxylic acid on the labelled antibody combining with the free amine groups on the modified electrode, there is a degree of uncertainty within our antibody-modified electrode system as to whether the antibody is positioned correctly for antigen binding at the paratopes.

The plethora of possibilities for non-optimal signal generation in this system means that the reliable and accurate sensing of antibody-antigen binding necessitates the use of a comparative approach (before and after exposure to antigen), rather than an absolute approach (where the system chemistry is only surveyed in the presence of a single concentration of antigen). Indeed, in our 
previous work [9], such antigen binding was inferred through the blocking (current reduction) of the ferrocene signal. In this work, we build upon the notions presented in our earlier work. However, we have been unable to quantify (1) the number of redox labels per antibody, (2) the yield of the antibody labelling process, and (3) the number of antibodies that are immobilised on the electrode surface and their orientation. We therefore are faced with the challenge of being unable to quantify the avidity of the monoclonal antibodies used, which impacts on our predictive optimisation of the electroimmunoassay.

Accordingly, we introduce a global blocking fraction, $\theta$, as a means to parameterise the amount of antigen on the electrode surface $(0 \leq \theta \leq 1)$. This is readily inferred from the kinetics of heterogeneous electron transfer in the presence and absence of antigen, as discussed by Savéant [32], and Compton and co-workers [33]: for an electrode that engages in an electron transfer process in the absence of any degree to which the redox process is blocked, with standard heterogeneous rate constant (in a Butler-Volmer framework) as $\mathrm{k}_{0}$, the rate constant $\left(\mathrm{k}_{\mathrm{s}}\right)$ is lowered in the presence of a process that impedes this. Such processes include partially blocking the active electrode area for a redox species that relies on transport to the electrode surface [34], or burying the redox-active group within a self-assembled monolayer so that the electron transfer kinetics are limited by the degree with which the counter ions may move through the structure [35]. It is this latter pathway that is believed to be the method through which the electroimmunoassay reported in our earlier work operates. Thus, to a first order approximation, we may write the following for the rate constant for electron transfer when antigen is bound to the antibody paratopes: $k_{s}=k_{0}(1-\theta)$. It thus follows that, provided there is a route for the measurement of the heterogeneous electron transfer rate constants, the degree of antigen binding may be quantified through equation (1):

$$
\theta=1-\frac{k_{s}}{k_{0}}
$$


This is a global antigen coverage parameter and is independent of the electrode architecture; it merely depends on the antigen binding thermodynamics and antigen-antibody on/off kinetics. We next consider the affinity of the proteinprotein interaction.

\section{Thermodynamics of Antigen-Antibody Recognition}

We consider the adsorption reaction between a freely diffusible antigen (AG) and a surface-immobilised receptor, such as a single paratope of an antibody (|-AB):

$$
|-A B+A G \rightleftarrows|-A B: A G
$$

Equality of the chemical potentials $\left({ }^{\mu}\right.$ ) of the antigen in both bulk solution and in the adsorbed states occurs at equilibrium,

$$
\mu_{A G}^{a d s}=\mu_{A G}^{b u l k}
$$

Thus, the change in standard Gibbs energy for the adsorption process, $\Delta \mathrm{G}^{0}{ }^{0}$, may be expressed in terms of the activities (a) of the solution and adsorbed antigen:

$$
a_{A G}^{a d s}=a_{A G}^{b u l k} e^{-\frac{\Delta G_{a d s}^{0}}{R T}}=a_{A G}^{b u l k} K_{a d s}
$$

where $\mathrm{R}$ is the molar gas constant, $\mathrm{T}$ is the absolute temperature and $\mathrm{K}_{\mathrm{ads}}$ is the equilibrium coefficient pertaining to the adsorption process. The adsorbed antigen activity may be expressed through the fraction of the blocked $(\theta)$ to unblocked $(1-\theta)$ active paratopes on the surface-confined antibody, yielding the familiar Langmuir isotherm, on incorporating solution activity effects into $\mathrm{K}_{\mathrm{ads}}$, to afford the Langmuir isotherm constant $\mathrm{K}^{\text {lang: }}$

$$
\frac{\theta}{1-\theta}=K^{\text {lang }} c_{A G}^{\text {bulk }}
$$

in which $\mathrm{c}^{\text {bulk }} \mathrm{AG}$ is the concentration of the antigen in bulk solution.

The Langmuir isotherm neglects, amongst other things, the presence of nearestneighbour interactions between adsorbed antigen molecules. The occurrence of such attractive or repulsive lateral interactions would result in a modification of the enthalpy change $\left(\Delta \mathrm{H}_{\mathrm{ads}}\right)$ associated with the adsorption equilibrium, and will 
depend on the fractional coverage. Thus, Taylor expansion of the standard enthalpy change for adsorption affords, to a first-order approximation:

$$
\Delta H_{a d s}=\Delta H_{a d s}^{0}(1-\alpha \theta)=\Delta G_{a d s}+T \Delta S_{a d s}
$$

where $\Delta \mathrm{G}_{\text {ads }}$ and $\Delta \mathrm{S}_{\text {ads }}$ respectively refer to the Gibbs energy change and the entropy change that take place on adsorption of the antigen, and $\alpha$ parameterizes the interactions: $\alpha<0$ corresponds to attractive interactions within the adsorbed layer so that a larger amount of heat is generated through successful adsorption; repulsive interactions are thus manifested through a reduced amount of liberated thermal energy on adsorption as $\alpha>0$.

Thus, the effect of accounting for lateral interactions is to correct the isotherm constant $\mathrm{K}^{\text {lang }}$ with:

$$
K=K^{\text {lang }} e^{A \theta}
$$

in which,

$$
A=\alpha \frac{\Delta H_{a d s}^{0}}{R T}
$$

so that the parameter $\mathrm{A}$ is negative for repulsive lateral interactions and positive attractive interactions as $\Delta H_{a d s}^{0}$ is negative. This gives rise to the Frumkin isotherm [36], which will be employed within this work:

$$
\frac{\theta}{1-\theta}=K^{\text {lang }} c_{A G}^{\text {bulk }} e^{A \theta}
$$

It follows that a plot of $\ln \left\{\frac{\theta}{c_{A G}^{\text {bulk }}(1-\theta)}\right\}$ against $\theta$ enables the determination of both the affinity constant $\mathrm{K}^{\mathrm{lang}}$ and the extent of lateral interactions (A). Note that since the adsorption process is exothermic (net bond forming), the constant A is expected to be positive for attractive interactions and negative for repulsive interactions.

We next examine the kinetics of the bio-recognition event. 
Rate Laws for the Formation of the Antibody-Antigen Complex from Freely Diffusible Antigen and Surface-Confined Antibody

We consider the adsorption reaction between a freely diffusible antigen (AG) and a surface-immobilised receptor, such as a single paratope of an antibody (|-AB):

$$
\left|-A B+A G \underset{k_{b}}{\stackrel{k_{f}}{\rightleftarrows}}\right|-A B: A G
$$

The rate at which the surface concentration $(\Gamma)$ of antibody and antibodyantigen complex changes via the heterogeneous adsorption kinetics balances the rate of antigen transport through diffusion-convection $[37,38]$ :

$$
\frac{d \Gamma_{\mid-A B: A G}}{d t}=k_{f} \Gamma_{1-A B} c_{A G}^{\text {surf }}-k_{b} \Gamma_{\vdash A B: A G} \simeq \frac{D}{\delta}\left(c_{A G}^{\text {bulk }}-c_{A G}^{\text {surf }}\right)
$$

in which $\mathrm{D}$ is the apparent diffusion coefficient of the antigen, with the concentrations of the antigen at the electrode surface and in bulk solution being described through the use of the superscripts surf and bulk, respectively. Note that although the experiments undertaken within this work (vide infra) are over long timescales and in quiescent solution, diffusion is considered here as being the major transport mechanism, since the natural convection that exists over this timescale may be treated in terms of an effective diffusion coefficient [39]. Thus, $\delta$ is the diffusion layer thickness, and the ratio $\mathrm{D} / \delta$ is the mass transport coefficient.

Mass balance on the electrode requires

$$
\Gamma_{T O T}=\Gamma_{1-A B}+\Gamma_{1-A B: A G}
$$

where $\Gamma_{\text {тот }}$ is the total surface concentration of the available paratopes. Using this definition, it therefore does not matter that we consider the overall reaction as being first order in antigen concentration, as we are not interested in the reaction avidity (the number of antigens that bind to each antibody), but rather, we are interested in the total number of available and active binding sites. In this way, we are able to treat the system as tantamount to adsorption to form a monolayer, even though the experimental system consisting of a platform made 
through diazonium reduction, is more than likely to be multi-layered (vide supra). Hence, denoting the fraction of blocked binding sites as

$$
\theta=\frac{\Gamma_{1-A B: A G}}{\Gamma_{T O T}}
$$

equations (11) to (13) afford the following for the surface concentration of antigen.

$$
c_{A G}^{\text {surf }}=\frac{\frac{D c_{A G}^{\text {bulk }}}{\Gamma_{T O T} \delta}+k_{b} \theta}{k_{f}(1-\theta)+\frac{D}{\Gamma_{T O T} \delta}}
$$

Thus, substitution into equation (11) yields:

$$
\frac{d \theta}{d t}=\frac{k_{f}(1-\theta) c_{A G}^{b u l k}-k_{b} \theta}{k_{f}\left(\frac{\Gamma_{T O T} \delta}{D}\right)(1-\theta)+1}
$$

In order to treat this particular problem in terms of general parameters, following Savéant [37], we define the characteristic diffusion time in equation (16)

$$
q=\frac{\Gamma_{T O T} \delta}{D c_{A G}^{b u l k}}
$$

and introduce the following dimensionless variables.

Normalised time:

$$
\tau=\frac{t}{q}
$$

Antibody-Antigen Complexation:

$$
\kappa=K^{\text {lang }} c_{A G}^{\text {bulk }}
$$

Ratio of adsorption to transport rates:

$$
\lambda=K^{\text {lang }} k_{b} c_{A G}^{\text {bulk }} q
$$

Hence, from equations (9) and (18), the ratio of blocked-to-unblocked paratope sites is given by

$$
\kappa e^{A \theta}=\frac{\theta}{1-\theta}
$$


It follows that the ratio of the forward to reverse rate constants is coveragedependent, q.v. equation (21),

$$
K^{\text {lang }} e^{A \theta}=\frac{k_{f}}{k_{b}}
$$

This means that one or both of $\mathrm{k}_{\mathrm{f}}$ and $\mathrm{k}_{\mathrm{b}}$ could be coverage-dependent. For simplicity, and in order to extract quantitative information from experimental data, we adopt the arbitrary convention that the rate constant for the adsorption process is coverage-dependent, whilst the desorption rate constant is independent of the coverage. Inasmuch as this is convenient, it can be partially rationalised as follows. First, if the electrode surface is intrinsically homogeneous and the concentration of antigen in the solution is fixed, the rate of antigen binding is dependent on the heterogeneous collision frequency $(\mathrm{Z})$, the fraction of available binding sites (1- $\theta)$, the fraction of antigen molecules that possess the activation energy for binding, $\mathrm{U}_{\text {bind, }}$ given by the Boltzmann factor $\exp \left(-\frac{U_{\text {bind }}}{R T}\right)$, and the condensation coefficient ("sticking" probability, $\mathrm{S}^{*}$ ), which is the fraction of moleculaes with energy $U>U_{\text {bind }}$ that adsorb. Thus, we may write the adsorption rate constant as $k_{f}=S^{*} Z \exp \left(-\frac{U_{\text {bind }}}{R T}\right)$. The rate constant for the desorption will be affected by the fraction of molecules possessing the activation energy for desorption ( $\left.\mathrm{U}_{\text {free }}\right)$ : $k_{b}=B \exp \left(-\frac{U_{\text {free }}}{R T}\right)$, where B is a constant. Since the heat of interaction, $\Delta H_{a d s}=U_{\text {bind }}-U_{\text {free }}$, is affected by the occurrence of attractive and repulsive lateral interactions, the rate constant ratio, $\frac{k_{f}}{k_{b}}=\frac{S^{*} Z}{B} \exp \left(-\frac{\Delta H_{a d s}}{R T}\right)$, would be independent of the antigen energy unless the sticking probability (and therefore $\mathrm{k}_{\mathrm{f}}$ ) in this formulation is more greatly affected by the statistics of the neighbouring environment than the constant B (and therefore $\mathrm{k}_{\mathrm{b}}$ ).

In terms of the dimensionless parameters, equation (15) becomes, 


$$
\frac{d \theta}{d \tau}=\frac{\lambda e^{A \theta}\left[1-\left(\frac{e^{-A \theta}+\kappa}{\kappa}\right) \theta\right]}{1+\lambda e^{A \theta}(1-\theta)}
$$

and which requires solution subject to the boundary condition

$$
\tau=0, \theta=0
$$

Note that this form of the rate law recognises that the extent of the lateral interactions within the adsorbed layer changes with time.

We first consider the case of the Langmuir isotherm $(A=0)$, for which no lateral interactions exist. This is the case that has been solved previously [37]. In brief, solution of the rate law proceeds through variable separation followed by integration through substitution of $1-\left(\frac{1+\kappa}{\kappa}\right) \theta$, to yield

$$
\lambda \theta-\left(1+\frac{\lambda}{1+\kappa}\right) \ln \left(1-\frac{1+\kappa}{\kappa} \theta\right)=\lambda\left(\frac{1+\kappa}{\kappa}\right) \tau
$$

This integrated rate law is for the general case, where activation and transport both control the bio-recognition process. If the rate constant for desorption is tiny and/or the characteristic diffusion time is small, viz. $k_{b} q \rightarrow 0$, so that the desorption process is slow compared with the transport rate (irreversible reaction), $\lambda \ll \kappa$. Furthermore, if the antigen concentration and/or the Langmurian binding constant is large, $1+\kappa \simeq \kappa$, equation (22) simplifies to $\frac{d \theta}{d \tau}=\frac{\lambda(1-\theta)}{1+\lambda(1-\theta)}$, which integrates to furnish equation (24).

$$
\theta-\frac{1}{\lambda} \ln (1-\theta)=\tau
$$

Equation (24) comprises two terms in the fractional coverage, which correspond to a linear dependence of coverage on antigen incubation time (for the larger values of $\lambda$, corresponding to faster transport than adsorption in the chemically irreversible binding scheme, equation (25)) which relaxes to the logarithmic form as $\lambda \rightarrow 0$ (corresponding to very slow desorption antigen desorption, equation (26)). 


$$
\begin{aligned}
& \theta \approx \tau \\
& \theta=1-e^{-\lambda \tau}
\end{aligned}
$$

We are interested in simplifying the rate law for the case that is likely to be most relevant to the experimental work, viz. the antibody-antigen binding is irreversible, but with $\theta \rightarrow 0$ and $\lambda$ « , so that the system is under activation control. Thus, equation (23) affords equation (27):

$$
-\ln \left(1-\frac{1+\kappa}{\kappa} \theta\right)=\lambda\left(\frac{1+\kappa}{\kappa}\right) \tau
$$

It is relatively facile to test this equation with the experimental data, since the equilibrium coverage of antigen is given by:

$$
\theta_{\text {eqm }}=\frac{\kappa}{1+\kappa}
$$

so that equation (27) may be recast as

$$
-\ln \left(1-\frac{\theta}{\theta_{\text {eqm }}}\right)=\frac{\lambda \tau}{\theta_{\text {eqm }}}=\frac{1}{\theta_{\text {eqm }}} K^{\text {lang }} k_{b} c_{A G}^{\text {bulk } t}
$$

Hence, a plot of $\left(1-\frac{\theta}{\theta_{\text {eqm }}}\right)$ against time enables the determination of the forward rate constant, as this is the product $\mathrm{K}^{\text {lang }} \mathrm{k}_{\mathrm{b}}$.

The observation of non-Langmuirian binding of labile antibodies to antigenmodified surfaces has been discussed within the literature [40]. For the case of the Frumkin isotherm considered in this work, $A \neq 0$, we have been unable to identify a method for the integration of the general case of the rate law, equation (22). Nevertheless, the approximation that $\kappa \gg e^{-A \theta}$ enables the rate law in equation (22) to yield the following tractable form.

$$
\frac{d \theta}{d t}=\frac{\lambda e^{A \theta}(1-\theta)}{1+\lambda e^{A \theta}(1-\theta)}
$$

Since from equation (7), $\mathrm{e}^{\mathrm{A}^{\theta}}$ corresponds to the ratio of the Frumkin binding constant $\left(\mathrm{K}\right.$, lateral interactions exist) to the Langmurian binding constant ( $\mathrm{K}^{\text {lang, }}$ no lateral interactions exist), using equation (18), the approximation made is 
that $K c_{A G}^{b u l k} \gg 1$. For repulsive lateral interactions, $\mathrm{A}$ is negative, and this approximation is only valid for small coverages (small antigen concentrations and/or small incubation times); for attractive lateral interactions, A is positive, and this approximation is only valid at large fractional coverage. Given that the Frumkin binding constant is not normally known for any particular set of experimental measurements, this approximation can be tested through its estimation via the (generally known) Langmuir binding constant of the untagged antibody.

Integration of equation (30) proceeds through variable separation and recognition of the validity of the power series expansion of $\mathrm{e}^{\mathrm{x}}$, to afford

$$
\lambda \theta+e^{-A}\left[-\ln (1-\theta)+\sum_{i=1}^{\infty} \frac{A^{i}}{i ! i}\left\{1-(1-\theta)^{i}\right\}\right]=\lambda \tau
$$

For the case when $\lambda \rightarrow 0$, corresponding to very slow desorption kinetics and/or faster diffusion to the modified surface relative to surface reaction, equation (31) may be written in the following form, from which kinetic information may be extracted from experimental data.

$$
e^{-A}\left[-\ln (1-\theta)+\sum_{i=1}^{\infty} \frac{A^{i}}{i ! i}\left\{1-(1-\theta)^{i}\right\}\right]=K^{\text {lang }} k_{b} c_{A G}^{\text {bulk } t}
$$

The sum-to-infinity in the expression on the left-hand side of equation (32) converges rapidly, with $\mathrm{i}=200$ being more than sufficient to ensure complete numerical convergence. Accordingly, rate constants may be extracted directly from experimental data in the light of equation (32) through plotting $e^{-A}\left[-\ln (1-\theta)+\sum_{i=1}^{\infty} \frac{A^{i}}{i ! i}\left\{1-(1-\theta)^{i}\right\}\right]_{\text {against time. }}$

We next apply these insights to experimental data. 


\section{Experimental}

\section{Chemical and Biochemical Reagents}

Human chorionic gonadotropin (hCG) reactivity: human $(100 \mu \mathrm{g})$ and mouse monoclonal anti-hCG: reactivity human, $500 \mu \mathrm{g}$ ), were purchased from Abcam (U.K.). The antigen, hCG was reconstituted to $1 \mathrm{~mL}$ with $10 \mathrm{mM}$ phosphate buffered saline solution (PBS) and stock solutions ranging $1 \mathrm{pg} \mathrm{mL}^{-1}$ to $1 \mu \mathrm{g} \mathrm{mL}^{-1}$ were prepared with $10 \mathrm{mM}$ PBS and $20 \mu \mathrm{L}$ aliquots stored in the freezer until use. 4-Nitrobenzene diazonium tetrafluoroborate was obtained from Sigma-Aldrich in the purest grade commercially available, and used as received. Tetrabutylammonium perchlorate (TBAP), ferrocenecarboxaldehyde (FcCHO), N,N-dimethylformamide (DMF), sodium borohydride $\left(\mathrm{NaBH}_{4}\right)$ and Tween 20 were obtained from Sigma-Aldrich. Water, with a resistivity of $18.2 \mathrm{M} \Omega \mathrm{cm}$, was obtained from Milli-Q Advantage A10 Water Purification System (Millipore, U.K.). Aqueous buffer solution was PBS at $\mathrm{pH}$ 7.4, prepared by dissolving one phosphate tablet (supplied from Sigma-Aldrich) containing $137 \mathrm{mM} \mathrm{NaCl}$, $2.7 \mathrm{mM} \mathrm{KCl}$ and $10 \mathrm{mM}$ phosphate in $200 \mathrm{~mL}$ deionised water. $\mathrm{N}$ hydroxysulfosuccinimide (sulfo-NHS, $2 \mathrm{mM}$ ) and N-(3-dimethylaminopropyl)-N'ethylcarbodiimide hydrochloride (EDC, $5 \mathrm{mM}$ ) were purchased from SigmaAldrich and both dissolved in $10 \mathrm{mM}$ PBS immediately prior to use. All other chemicals were purchased from Sigma-Aldrich (U.K.) in the highest purity grade commercially available and used without further purification.

\section{Instrumentation}

Electrochemical studies were performed in a modified three-electrode set-up with a tin-doped indium oxide (ITO) coated glass working electrode (CB-50INCUV, sheet resistance 5-15 $\Omega$, dimensions $7 \times 50 \times 0.7 \mathrm{~mm}$, Delta Technologies Ltd., U.S.A.). A defined area was prepared with Fisherclark WS5002 self adhesive paper reinforcement rings (determined using a travelling microscope to be of area $0.28 \mathrm{~cm}^{2}$ ), with one end used for electrical connection via application of copper tape. Up to three individual electrode areas were prepared per electrode. Owing to the increase in the electrode resistance with distance from the copper tape connection, the location of the three working areas of the single ITO were each precisely measured and the resistance from the copper tape connector to 
the centre of each area determined with a digital voltmeter. This was undertaken so that in experiments where inter-electrode comparisons needed to be made, only those working areas that had the same electrode resistance were used. It was observed that the area closest to the copper tape connector had a resistance of $0.37 \pm 0.01 \mathrm{k} \Omega$; the next closest area had a resistance of $0.57 \pm 0.01 \mathrm{k} \Omega$; the furthest away area had a resistance of $0.82 \pm 0.05 \mathrm{k} \Omega$. The determined resistivity (measured as resistance multiplied by electrode area and normalised by the straight-line surface distance from the centre of the area to the copper contact) was observed to be constant over the three areas, at $2.56 \pm 0.08 \mu \Omega \mathrm{m}$.

Electrochemical measurements were made in a $30 \mu \mathrm{L}$ sample droplet with Pt or nichrome wire as the counter electrode and a standard calomel electrode (SCE, 3 $\mathrm{M} \mathrm{KCl)} \mathrm{reference} \mathrm{electrode} \mathrm{(BAS,} \mathrm{U.K.).} \mathrm{All} \mathrm{potentials} \mathrm{are} \mathrm{reported} \mathrm{here} \mathrm{against}$ this reference, unless otherwise stated. Measurements were controlled by a $\mu$ AUTOLAB-type-III potentiostat. Cyclic voltammetry (CV) of the modified electrodes was performed over variable scan rates between $0.02 \mathrm{~V} \mathrm{~s}^{-1}$ to $1 \mathrm{~V} \mathrm{~s}^{-1}$. The scan rates were varied in a randomised manner so as to guard against electrode history effects. All electrochemical investigations were undertaken at ambient temperature $(293 \pm 2 \mathrm{~K})$, in laboratory air of relative humidity in the range $50-70 \%$.

\section{ITO Surface Modification with Amine Groups}

The working electrode employed for this study was made from ITO, because these electrodes exhibited the most reproducible signals for this type of surfacebased electrochemistry, compared with glassy carbon or polycrystalline gold. ITO electrodes were cleaned prior to modification with isopropanol. Amine modification of the electrode surface was achieved through electrochemical reduction of $2 \mathrm{mM}$ 4-nitrobenzene diazonium tetrafluoroborate in 0.1 M TBAP$\mathrm{MeCN}$ for the period of four reduction-oxidation cycles ( $+0.7 \mathrm{~V}$ to $-0.4 \mathrm{~V})$ to yield a nitrobenzene-modified electrode, followed by six-electron, six-proton reduction to afford the amine, aqueous ethanol containing $0.1 \mathrm{M} \mathrm{KCl}(10: 90, v / v)$ 
from $+0.4 \mathrm{~V}$ to $-1.25 \mathrm{~V} v s$. SCE through four redox cycles, in a manner reported previously for gold electrodes [9].

\section{Synthesis of Ferrocene-Labelled Antibodies}

Anti-hCG antibodies were chemically labelled with ferrocene through the coupling scheme reported previously [9]: $10 \mu \mathrm{L}$ mouse monoclonal IgG was diluted to $200 \mu \mathrm{L}$ with $10 \mathrm{mM}$ PBS (pH 7.4) and the $\mathrm{pH}$ adjusted to $\mathrm{pH}$ (9 by addition of 5 wt.\% potassium carbonate, with subsequent dilution with a $100 \mathrm{mg} \mathrm{mL}^{-1}$ solution of ferrocene carboxaldehyde (FcCHO) in DMF, such that the FcCHO was in excess. This was followed by incubation at ambient temperature for $30 \mathrm{~min}$ to form the imine, subsequently reduced with sodium borohydride ( $1.0 \mathrm{mg}$ ), and incubated at ambient temperature for $10 \mathrm{~min}$ in order to form the secondary amine (Scheme 1 ). The solution was adjusted to $\mathrm{pH} \sim 7.3$ using 1.0 M HCl. Excess tag and solvent were removed from the ferroceneconjugated antibody product after reaction by centrifugation and washed with $10 \mathrm{mM}$ PBS (12 krpm for $20 \mathrm{~min}$, followed by twice washing with PBS at $12 \mathrm{krpm}$ for 5 mins, using Vivaspin 500 centrifugal filters purchased from GE Healthcare, Sweden). Ultimately, $400 \mu \mathrm{L}$ of the purified and concentrated ferroceneconjugated antibody was obtained and stored within a refrigerator at $277 \mathrm{~K}$ until immediately prior to use.

\section{Bio-Conjugation of Ferrocene-Labelled Antibodies to ITO Surface}

The immobilisation of ferrocene-tagged antibody on the electrode surface was achieved through the coupling of carboxylic acid groups of the antibody to the primary amine groups of the phenylamine-modified ITO electrode surface via an amide linkage, in a manner reported previously [9]. The $400 \mu \mathrm{L}$ aliquot of ferrocene-conjugated antibody was mixed with the same volume of activation buffer ( $2 \mathrm{mM}$ EDC and $5 \mathrm{mM}$ sulfo-NHS in $10 \mathrm{mM}$ PBS) for $15 \mathrm{~min}$ to activate the carboxy terminal of the antibody. Subsequently, $30 \mu \mathrm{L}$ of this solution was applied to the amine functionalized ITO electrode and stored at $277 \mathrm{~K}$ for 18 hours, after which the electrodes were washed with 0.1 vol.\% Tween 20 in 10 mM PBS, followed by PBS to remove unbound antibody, before drying under a 
stream of nitrogen. The electrodes were used immediately, or stored in $10 \mathrm{mM}$ PBS at $277 \mathrm{~K}$ until use. Typical storage times ranged between 24 - $48 \mathrm{~h}$.

\section{Hormone Measurement}

First, any non-specific binding sites on the modified ITO surface were blocked by the addition of $20 \mu \mathrm{L}$ of 1 wt. \% BSA-10mM PBS, pH 7.0 for $30 \mathrm{~min}$ at room temperature. Following this, the modified electrodes were rinsed with 0.1 vol.\% Tween 20 in $10 \mathrm{mM}$ PBS followed by PBS. All electrodes were then tested in physiological PBS using cyclic voltammetry for a series of ten scan cycles at $0.1 \mathrm{~V} \mathrm{~s}^{-1}$ to ensure stability of the surface and of the corresponding signal. This was followed by voltammetry at a variable scan rate (v) within the range $0.02 \leq \mathrm{v} / \mathrm{V} \mathrm{s}^{-1} \leq 1.0$, to obtain blank calibration data. After this, the antibodymodified electrodes were incubated in known concentrations of hCG antigen in $10 \mathrm{mM}$ PBS for particular time periods $(15,30$ and $60 \mathrm{~min})$ at room temperature. Since the loss of pure bulk water to dry air occurs at an evaporation rate of $5.5 \mu \mathrm{g} \mathrm{s}^{-1}$ at $298 \mathrm{~K}$ [41], the incubations with antigen were undertaken by spreading antigen solution over the ITO slide. In this way, evaporation of water from the exposed antigen solution, driven by the water vapour concentration difference from $0.58 \mathrm{mM}$ (at $60 \%$ relative humidity) to $0.96 \mathrm{mM}$ (at $100 \%$ relative humidity), would be sufficiently neglible. After incubation, the electrodes were rinsed with Tween 20 and PBS, followed by drying in a nitrogen stream, to remove any loosely bound antigen. The electrodes were then subject to re-examination by variable scan rate cyclic voltammetry in PBS, with scan rates within the range $0.02 \leq \mathrm{v} / \mathrm{V} \mathrm{s}^{-1} \leq 1.0$.

Fitting of Experimental Data to Determine the Global Antigen Coverage Parameter For a one-electron process occurring within a surface monolayer, the current that results from non-interacting redox centres, is the summation of oxidation and reduction processes:

$$
i=F S\left(k^{o x} \Gamma^{r e d}-k^{r e d} \Gamma^{o x}\right)
$$


where $\mathrm{i}$ is the current, $\mathrm{S}$ the electrode area, $\mathrm{F}$ the Faraday constant and with rate constants $\mathrm{k}^{\mathrm{j}}$ and surface concentrations $\Gamma^{\mathrm{j}}(\mathrm{j}=\mathrm{ox}$ or red), with the superscript identifying the particular redox state. When electron transfer occurs from the Fermi energy of the electrode, and the symmetry factor is close to $1 / 2$, it has been demonstrated that the Butler-Volmer formulation for electrode kinetics is adequate [35]. This latter formulation of the electrode kinetics readily empowers the determination of the standard heterogeneous rate constant for oxidation of the immobilised ferrocenes $\left(\mathrm{k}_{\mathrm{s}}\right)$ to be extracted from the variation of the peak oxidation potential with scan rate, since:

$$
\begin{aligned}
& k^{o x}=k_{s} \exp \left\{-\frac{F}{2 R T}\left(E-E^{0^{\prime}}\right)\right\} \\
& k^{\text {red }}=k_{s} \exp \left\{\frac{F}{2 R T}\left(E-E^{0^{\prime}}\right)\right\}
\end{aligned}
$$

where $\mathrm{F}$ is the Faraday constant $\left(96484.6 \mathrm{C} \mathrm{mol}^{-1}\right), \mathrm{R}$ is the molar gas constant (8.3154 $\left.\mathrm{J} \mathrm{mol}^{-1} \mathrm{~K}^{-1}\right), \mathrm{T}$ is the absolute temperature and $\left(\mathrm{E}^{-\mathrm{E}^{0}}\right)$ is the applied overpotential. Accordingly, the experimental data were treated simplistically, so that values of $\mathrm{k}_{\mathrm{s}}$ were extracted from the variation of peak potential with scan rate upon solving equation (33) numerically under voltammetric conditions, without considering any Ohmic loss. The variable scan rate data taken in the absence of antigen enables the determination of $\mathrm{k}_{0}$, since antigen blocking fraction $(\theta)$ is zero, and thus $\mathrm{k}_{\mathrm{s}}=\mathrm{k}_{0}$. Thus, in the presence of the antigen, the value of $\mathrm{k}_{\mathrm{s}}$ relates to the extracted value of $\mathrm{k}_{0}$ through $k_{s}=k_{0}(1-\theta)$, allowing the global blocking fraction $(\theta)$ to be inferred at each incubation time via comparison between blocked and unblocked electrode responses.

It is important to realise that, since the experiments were undertaken with no correction for Ohmic loss, the values of $\mathrm{k}_{\mathrm{s}}$ (and $\mathrm{k}_{0}$ ) estimated via this protocol necessarily reflect contributions from both electrode kinetics and electrode/solution resistances. Here, given that ferrocene redox tags were employed, and that the solution resistance is constant between experiments, the ITO electrode resistance is the largest source of error. This means that only those working electrodes areas that held the same resistance could be compared in 
this manner. This is fully correct, since only those signals, which derived from electrodes with similar resistances, were compared to obtain the results reported herein. It also identifies that the values of $\mathrm{k}_{0}$ and $\mathrm{k}_{\mathrm{s}}$ extracted from the data are meaningless, unless directly compared. 


\section{Results and Discussion}

Figure 2 illustrates the voltammetry of anti-hCG antibody-modified electrodes in PBS at a scan rate of $0.1 \mathrm{~V} \mathrm{~s}^{-1}$, over ten consecutive cycles. As expected for an electrode modified with soft matter $[35,42,43]$, the first few cycles correspond to "break-in" scans, with significant capactative contributions owing to the molecular rearrangements occurring so that counter ions to the immobilised, electrogenerated ferricinium ions may find pathways through the protein structure on the electrode surface. Consecutive cycling enables the voltammetric waveshape to stabilise into the well-defined waves at $E_{\text {mid }}=1 / 2\left(E_{p} 0 x+E_{p} R e d\right)=$ $0.22 \mathrm{~V}$ vs. SCE, which correspond to the oxidation and re-reduction of the ferrocene labels covalently bound onto the ITO electrode. Increasing the voltammetric scan rate results in larger separation between the peak potentials. This is expected, since the heterogeneous rate constant for long-range electron transfer through a peptide chain is [44] $0.2-0.5 \mathrm{~s}^{-1}$ for peptides comprising 6-11 amino acids; in larger nano-objects, such as the antibody proteins, it is expected that electron transfer rate constants would be considerably smaller. Figure 3 illustrates typical data for the oxidation peak potential. The fitting of the trend in these data with experimental timescale with those anticipated from the ButlerVolmer theory, by finding the minimum in the mean absolute error between experiment and theory, enables the determination of the rate constant for the set of data.

In order to determine the global fractional antigen coverage, $\theta$, the heterogeneous electron transfer rate constant, $\mathrm{k}_{\mathrm{s}}$ is determined for both the 'blank' electrode (prior to antigen incubation) and post incubation, as required by equation (1). By varying the timescale of the antigen incubation period, and by varying the antigen concentration, both the thermodynamics and kinetics of the antibody-antigen binding event may be unravelled. Figure 4 shows the timedependence of the blocking of the antibody-modified electrode in the presence of hCG of various concentrations. As expected, as the concentration of antigen increases, the surface coverage also increases until a point of equilibrium, or steady-state, is reached. Langmurian plots of $\theta^{-1}$ against $\left(c_{A G}^{\text {bulk }}\right)^{-1}$ afforded highly 
curved plots of the form $\frac{1}{\theta}=0.0775\left(\frac{1}{c_{A G}^{b u l k}}\right)^{0.1691}$ correlation coefficient of 0.925 , and with greatest deviations at low antigen concentrations. This is an indication of non-Langmurian binding. Thus, at equilibrium $(\mathrm{t}=60 \mathrm{~min})$, a plot of $\ln \left\{\frac{\theta}{c_{A G}^{\text {bulk }}(1-\theta)}\right\}$ against $\theta$, as suggested by equation (9), and depicted in Figure 5, enables the deduction of the affinity constant, $\mathrm{K}^{\text {lang }}=3.9 \pm 0.8 \times 10^{11} \mathrm{M}^{-1}$. This is, in fact, two orders of magnitude larger than that reported for the non-redox tagged antibody [45], $2.9 \times 10^{9} \mathrm{M}^{-1}$, suggesting that the presence of the hydrophobic redox tag empowers additional interactions with the antigen, and which augers well for an analytical protocol using this modified antibody. The gradient of the plot in Figure 5 enables the deduction of the Frumkin isotherm parameter: $A=-14.4 \pm 1.3$. The negative value of this is commensurate with repulsive interactions between chemisorbed antigens, as expected for a surface where steric crowding may occur.

In order to determine the effect of the larger affinity constant on the rate constants for the adsorption and desorption of the antigen, experimental data were treated through the plots suggested by equation (32). From the gradients of these plots (not shown), the rate constant for antigen desorption was identified as being essentially independent of the antigen concentration: $\mathrm{k}_{\mathrm{b}}=6.5 \pm 2.7 \times 10^{-5} \mathrm{~s}^{-1}$, enabling the rate constant for antigen adsorption to be determined from equation (21): $\mathrm{k}_{\mathrm{f}}=2.2 \pm 0.9 \times 10^{7} \exp (-\{14.4 \pm 1.3\} \theta) \mathrm{M}^{-1} \mathrm{~s}^{-1}$. It is notable that these data are consistent with the increase in favourable binding interactions; the rate constant for adsorption is, at zero coverage, an order of magnitude larger than that of the untagged antibody, $4.0 \times 10^{6} \mathrm{M}^{-1} \mathrm{~s}^{-1}$ [45], whilst that for desorption is an order of magnitude smaller than that of the unlabelled antibody, $1.4 \times 10^{-4} \mathrm{~s}^{-1}$ [45], suggesting that the hydrophobic redox tag (ferrocene) may promote the binding process.

In order to examine the closeness of fit between the experimental data and those that may be theoretically observed based on the average kinetic data obtained, 
equation (22) was simulated in Matlab (version R2013a) using the finite difference method. In this case, first the parameter $\mathrm{q}$ (the characteristic diffusion time) given by equation (16) was identified though a minimum deviation between experiment and theory with its value being varied between 1 and $4000 \mathrm{~s}$, with a convergent data observed for a time node of $10^{-2} \mathrm{~s}$. Typical values of q were between 1-48 min (25 min average), suggesting that above this time, antigen transport to the surface may start to dominate the response. There was no apparent correlation of q with hCG concentration, except that at lower hCG concentrations $\mathrm{q}$ was towards the lower limit, and at higher hCG concentrations q was towards the higher limit. Knowledge of the optimised $q$ values enabled the best-fit data to be determined and contrasted with the experimental points, as indicated in Figure 6. It is readily seen that whilst the fits are reasonable for lower concentrations of the antigen, the overall fit worsens at higher antigen concentrations. Since rate constants were estimated from activation approximations (equation (32)), and the solid lines fits in Figure 6 determined through simulation of equation (22), which includes transport processes, the poorer fit of the experimental transients at higher antigen concentration corresponds to transport-dominated processes at shorter incubation times. This intuitive result nevertheless identifies that the protocols developed may be exploited for bioanalytical measurement of antigen concentration at short incubation times and for low antigen concentrations.

In the light of these data, it follows that the use of low antigen concentrations with short ( $15 \mathrm{~min})$ modified electrode-antigen incubations are sufficient for this type of platform to be used for assessing the amount of hCG within a PBS solution. Accordingly, in order to demonstrate a quantitative analytical protocol of hCG, working curves for each respective incubation time were obtained for the clinically relevant hCG concentration range within PBS solution. These comprise plots of $\theta$ vs. $\lg \left([\mathrm{hCG}] / \mathrm{ng} \mathrm{mL} \mathrm{L}^{-1}\right)$, and are illustrated in Figure 7. Such curves are linear with $\mathrm{R}^{2}$ values greater than 0.97 (over three repeats), verifying that a $15 \mathrm{~min}$ incubation time is suitable for rapid and quantitative analysis, with excellent stability and inter-experiment reproducibility. Limits of detection (LoD) were determined (as three times the standard deviation normalised by the 
calibration graph gradient) to be $20.2,28.3$ and $19.8 \mathrm{mIU} \mathrm{mL}^{-1}$ for the 15,30 and 60 min incubation times, respectively, for hCG concentrations in the range $100 \mathrm{pg} \mathrm{mL}^{-1}$ to $1 \mu \mathrm{g} \mathrm{mL}^{-1}$. It is notable that the LoD for the $15 \mathrm{~min}$ incubation is around the $25 \mathrm{mIU} \mathrm{mL}^{-1}$ cut-off considered for pregnancy testing of urine hCG for a gestational age of around four weeks, identifying clinical viability of this protocol.

\section{Conclusions}

This work has considered the chemical biophysics underpinning an electrochemical approach which monitors hGC binding to monoclonal antibodies immobilised through a molecular wire-type platform on an electrode surface. It has been observed that the binding is chemically irreversible and nonLangmurian, as significant repulsive lateral interactions exist during the chemoreception event. The kinetics of the binding, coupled with the larger value of the Langmurian affinity constant in the redox-tagged antibody compared with the untagged literature data, suggest that the chemical nature of the redox tag on the antibody (in this case, ferrocene) may actually enable slower dissociation of the antibody-antigen complex, with coverage-dependent binding kinetics. Through the comparison of experimental data treated under activation control and non-Langmuirian irreversible binding with a simulation of rate law under no such assumptions, it has been observed that the approximations hold for low concentrations of antigen, as expected, even for short antigen-antibody incubation times. This has been validated experimentally, and the potential use of this methodology for rapid and quantitative electroimmunoassay demonstrated.

\section{Acknowledgements}

This work was supported by BBSRC (GR/N BB/G017042/1) and EPSRC (GR/N EP/G020833/1). LIP thanks both the Hull-York Medical School and the Department of Chemistry, University of Hull for additional financial support. 


\section{References}

1. See, for example, D. Voet, J. G. Voet, C. W. Pratt, Fundamentals of Biochemistry, upgrade edn., John Wiley, New Jersey, 2002.

2. See, for example, D. Male, J. Brostoff, D. B. Roth, I. Roitt, Immunology, $7^{\text {th }}$ edn., Elsevier, Philadelphia, 2006.

3. C. Demaille, J. Moiroux, J.-M. Saveant, C.Bourdillon, in H. Mohwald, Y. Lvov (eds.), Protein Architecture Interfacing Molecular Assemblies and Immobilisation Biotechnology, Marcel Dekker, New York, 1999, p.311.

4. H. C. Berg, E. M. Purcell, Biophys. J., 1977, 20, 193.

5. V. Gubala, L. F. Harris, A. J. Ricco, M. X. Tan, D. E. Williams, Anal. Chem., 2012, 84, 487.

6. E. A. Newsholme, A. R. Leech, Biochemistry for the Medical Sciences, John Wiley, Chichester, 1983.

7. J. Ruzicka, J. Stary, Atomic Energy Rev., 1964, 2, 3.

8. See, for example, J. Yakovleva, J. Emnéus, in P. N. Bartlett (ed.), Bioelectrochemistry: Fundamentals, Experimental Techniques and Applications, John Wiley, Chichester, 2008.

9. Y.-H. Dou, S. J. Haswell, J. Greenman, J. Wadhawan, Electroanalysis, 2012, 24, 264.

10. L. A. Cole, Clin. Chim. Acta, 2012, 413, 48.

11. L. A. Cole, Clin. Chem., 1997, 43, 12.

12. T. Chard, Human Reprod., 1992, 7, 701.

13. C. Chandrasekhar, Clin. Imag., 2008, 32, 468.

14. C. L. Buckner, L. Wilson, C. N. Papadea, Ann. Clin. Lab. Sci., 2007, 37, 186.

15. U.-H. Stenman, A. Tiitinen, H. Alfthan, L. Valmu, Human Reprod. Update, 2006, 12, 769.

16. R. S. Berkowitz, D. P. Goldstein, New Engl. J. Med., 1996, 335, 1740.

17. Great Ormond Street Hospital for Children NHS Foundation Trust, Routine Pregnancy Testing Before Treatment, GOSH NHS Foundation Trust, London, Ref. 2013F1626, 2014.

18. J. Lei, S. Mei, Y. Zhou, T. Jing, J. Chin. Chem. Soc., 2014, 61, 638.

19. J. T. Liu, R. P. Liu, M. X. Wang, C. X. Liu, J. P. Luo, X. X. Cai, Chin. J. Anal. Chem., 2009, 37, 985.

20. D. Liu, F. Wu, C. Zhou, H. Shen, H. Yuan, Z. Vu, L. Ma, L .S. Li, Sens. Acts. B, Chem., 2013, 186, 235.

21. R. Li, D. Wu, H. Li, C. Xu, H. Wang, Y. Zhao, Y. Cai, Q. Wei, B. Du, Anal. Biochem., 2011, 414, 196.

22. N. X. Viet, M. Chikae, Y. Ukita, K. Maehashi, K. Matsumoto, E. Tamiya, P. H. Viet, Y. Takamura, Biosens. Bioelectron., 2013, 42, 592.

23. S. Teixeira, R. S. Conlan, O. J. Guy, M. G. F. Sales, Electrochim. Acta, 2014, 136, 323.

24. X. Li, S. Weng, B. Ge, Z. Yao, H. Z. Yu, Lab Chip, 2014, 14, 1686.

25. S. A. Butler, S. A. Khanlian, L. A. Cole, Clin. Chem., 2001, 47, 2131.

26. L. V. Furtado, C. M. Lehman, C. Thompson, D. G. Grenache, Am. J. Clin. Pathol., 2012, 137, 201.

27. M. M. L. M. Vareiro, J. Liu, W. Knoll, K. Zak, D. Williams, A. T. A. Jenkins, Anal. Chem., 2005, $77,2426$.

28. H. Xu, J. R. Liu, D. E. Williams, J. Phys. Chem. B, 2006, 110, 1907.

29. H. Xu, X. Zhao, J. R. Lu, D. E. Williams, Biomacromolec., 2007, 8, 2422.

30. S. Munteanu, J.-P. Roger, Y. Fedala, F. Amiot, C. Combellas, G. Tessier, F. Kanoufi, Faraday Discuss., 2013, 164, 241.

31. A. J.Wain, N. S. Lawrence, P. R. Greene, J. D. Wadhawan, R. G. Compton, Phys. Chem. Chem. Phys., 2003, 5, 1867.

32. C. Amatore, J.-M. Savéant, D. Tessier, J. Electroanal. Chem., 1983, 147, 39.

33. B. A. Brookes, T. J. Davies, A. C. Fisher, R. G. Evans, S. J. Wilkins, K. Yunus, J. D. Wadhawan, R. G. Compton, J. Phys. Chem. B, 2003, 107, 1616.

34. T. J. Davies, B. A. Brookes, A. C. Fisher, K. Yunus, S. J. Wilkins, P. R. Greene, J. D. Wadhawan, R. G. Compton, J. Phys. Chem. B, 2003, 107, 6431.

35. C. Amatore, E. Maisonhaute, B. Schollhorn, J. Wadhawan, ChemPhysChem, 2007, 8, 1321.

36. See, for example, B. B. Damaskin, A. N. Frumkin, in N. S. Hush (ed.), Reactions of Molecules at Electrodes, John Wiley, London, 1971, p.1. 
37. C. Demaille, J. Moiroux, J.-M. Saveant, C.Bourdillon, J. Am. Chem. Soc., 1999, 121, 2401.

38. I. Bhugun, F. C. Anson, J. Electroanal. Chem., 1997, 439, 1.

39. C. Amatore, C. Pebay, L. Thouin, A. Wang, J.-S. Warkocz, Anal. Chem., 2010, 82, 6933.

40. D. B. Hibbert, J. J. Gooding, P. Erokhin, Langmuir, 2002, 18, 1770.

41. K. J. Beverley, J. H. Clint, P. D. I. Fletcher, Phys. Chem. Chem. Phys., 1999, 1, 909.

42. R. W. Murray, in R. W. Murray (ed.), Molecular Design of Electrode Surfaces, John Wiley, New York, 1992, p.1.

43. A. J. Wain, J. D. Wadhawan, R. G. Compton, ChemPhysChem, 2003, 4, 974.

44. B. R. Chaudhry, J. D. E. T. Wilton-Ely, A. B. Tabor, D. J Caruana, Phys. Chem. Chem. Phys., 2010, 12, 9996.

45. http://www.abcam.com; accessed June 27, 2016. 


\section{Figure Legends}

\section{Scheme 1}

Schematic illustration depicting the synthesis of the ferrocene tagged antibodies. In panel (a), ferrocenecarboxyaldehyde is initially attached to the antibody through a Schiff base; panel (b) identifies the conditions for the conversion of the Schiff base to form the ferrocene-conjugated antibody employed within this work.

\section{Figure 1}

Cartoon illustrating the nature of the electrochemical responses considered within this manuscript. Although the electrode surface is shown as a monolayer in this illustration, it is recognised that this is likely not the case (see text).

\section{Figure 2}

Cyclic voltammograms $\left(0.1 \mathrm{~V} \mathrm{~s}^{-1}\right)$ of the ferrocene-tagged antibody immobilised on an ITO electrode and immersed in $10 \mathrm{mM}$ PBS at pH 7.4. Ten scans are shown, testifying to the stability of the modified electrode within this medium.

\section{Figure 3}

Extraction of the heterogeneous rate constants for the oxidation of the ferrocene redox tag from variable scan rate cyclic voltammograms undertaken in $10 \mathrm{mM}$ PBS at $\mathrm{pH}$ 7.4. Experimental data are illustrated as blue circles; the solid line corresponds to the expected behaviour under ButlerVolmer kinetics, with E representing the peak oxidative potential and $\mathrm{v}$ the voltammetric sweep rate. The insert corresponds to the voltammograms analysed, with the legend affording the scan rates in $\mathrm{mV} \mathrm{s}^{-1}$. In these experiments, the variable scan rate started from $0.1 \mathrm{~V} \mathrm{~s}^{-1}$ and varied in a randomised fashion, returning to $0.1 \mathrm{~V} \mathrm{~s}^{-1}$ at the end, so as to encompass any effects due to ferricinium ion instability within this medium.

\section{Figure 4}

Plots illustrating the global blocking coverage $(\theta)$ as a function of antigen-incubation time in 10 $\mathrm{mM}$ PBS at pH 7.4, with varying concentrations of hGC in the range $10^{-1} \leq[\mathrm{hCG}] / \mathrm{ng} \mathrm{mL}^{-1} \leq 10^{3}$. Key: The individual hCG concentrations are: $100 \mathrm{pg} \mathrm{mL}^{-1}(\mathrm{o}), 500 \mathrm{pg} \mathrm{mL}^{-1}(\mathrm{x}), 1 \mathrm{ng} \mathrm{mL}^{-1}(+), 2$ ng $\mathrm{mL}^{-1}\left(^{*}\right), 5 \mathrm{ng} \mathrm{mL} \mathrm{L}^{-1}(\square), 10 \mathrm{ng} \mathrm{mL}^{-1}(\diamond), 20 \mathrm{ng} \mathrm{mL}^{-1}(\nabla), 100 \mathrm{ng} \mathrm{mL}^{-1}(\Delta)$ and $1 \mu \mathrm{g} \mathrm{mL}^{-1}$ (pentagrams).

\section{Figure 5}

Typical analysis of the antigen-antibody binding evolution for hCG under the Frumkin adsorption isotherm equation (9). The data are shown for three incubation times: 15 min (blue diamonds), $30 \mathrm{~min}$ (green triangles) and $60 \mathrm{~min}$ (red squares). Note that the extraction of thermodynamic parameters is only valid for the data from $60 \mathrm{~min}$ as this corresponds to the point of equilibrium.

\section{Figure 6}

Comparison of experiment data (open points) with theory (solid lines) for the evolution of the fractional coverage of the antibody-modified electrode surface, during the course of antigen incubation. Theoretical data were obtained through simulation of equation (22) using the parameters: $\quad \mathrm{A}=-14.4 \pm 1.3 ; \mathrm{K}^{\text {lang }}=3.9 \pm 0.8 \times 10^{11} \mathrm{M}^{-1} ; \mathrm{k}_{\mathrm{b}}=6.5 \pm 2.7 \times 10^{5} \mathrm{~s}^{-1}$. The data correspond to hCG concentrations of $100 \mathrm{pg} \mathrm{mL}^{-1}$ (blue lines and circles), $500 \mathrm{pg} \mathrm{mL}^{-1}$ (red lines and circles), $1 \mathrm{ng} \mathrm{mL}^{-1}$ (black lines and circles), $2 \mathrm{ng} \mathrm{mL}-1$ (green lines and circles), $5 \mathrm{ng} \mathrm{mL}^{-1}$ (magenta lines and circles), $10 \mathrm{ng} \mathrm{mL}^{-1}$ (cyan lines and circles), $20 \mathrm{ng} \mathrm{mL}^{-1}$ (yellow lines and circles), $100 \mathrm{ng} \mathrm{mL}^{-1}$ (red lines and squares) and $1 \mu \mathrm{g} \mathrm{mL}^{-1}$ (blue lines and squares). 


\section{Figure 7}

Analytical calibration plot of fractional hCG coverage on the antibody-modified electrode extracted after hCG incubation times of 15, 30 and 60 min periods in $10 \mathrm{mM}$ PBS at pH 7.4, with hCG concentration. Lines of optimum fit are provided, for the data (averaged over three repeats). 
Scheme 1

(a)

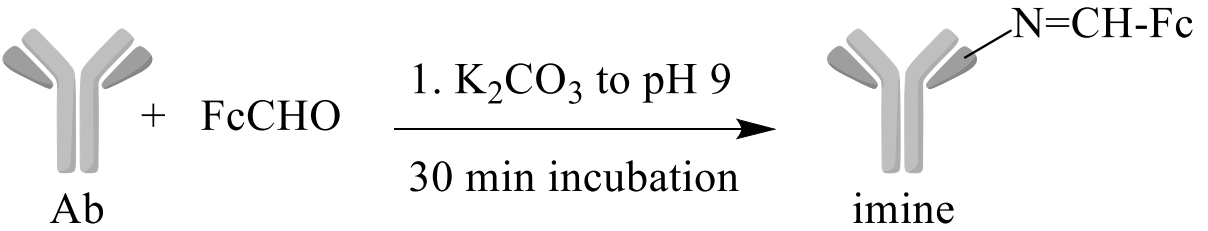

(b)
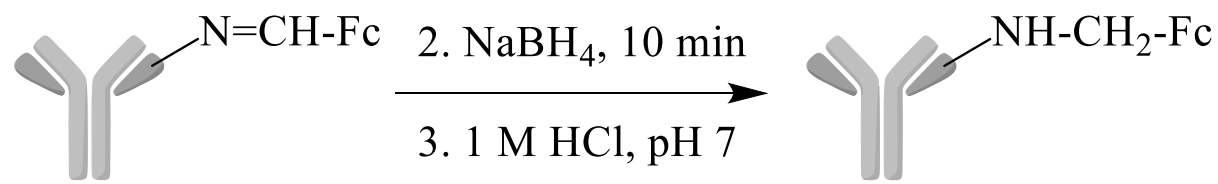

final tagged

product 
Figure 1

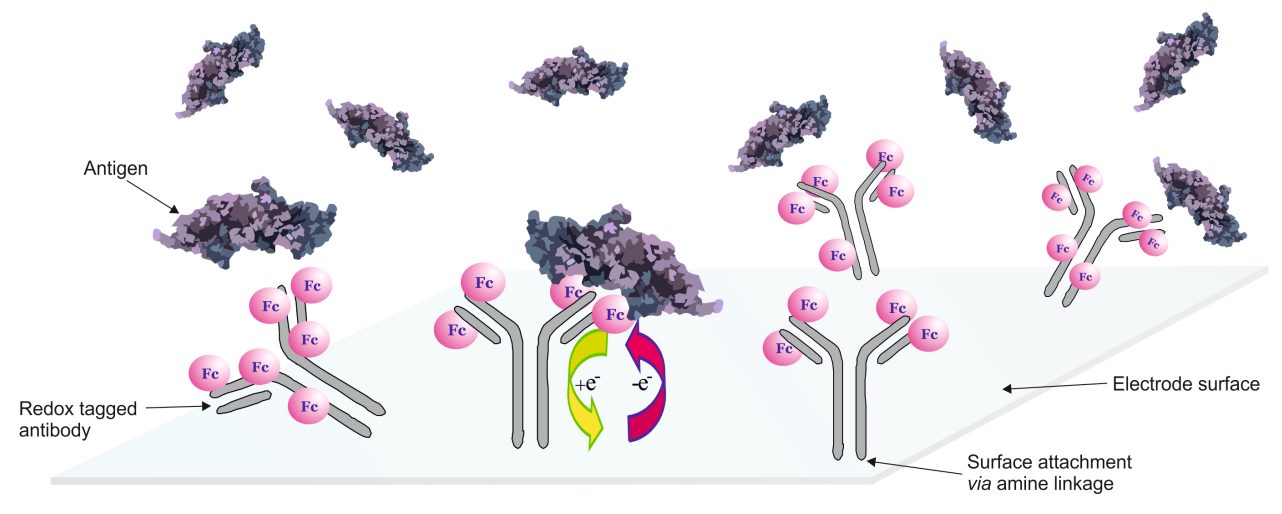


Figure 2

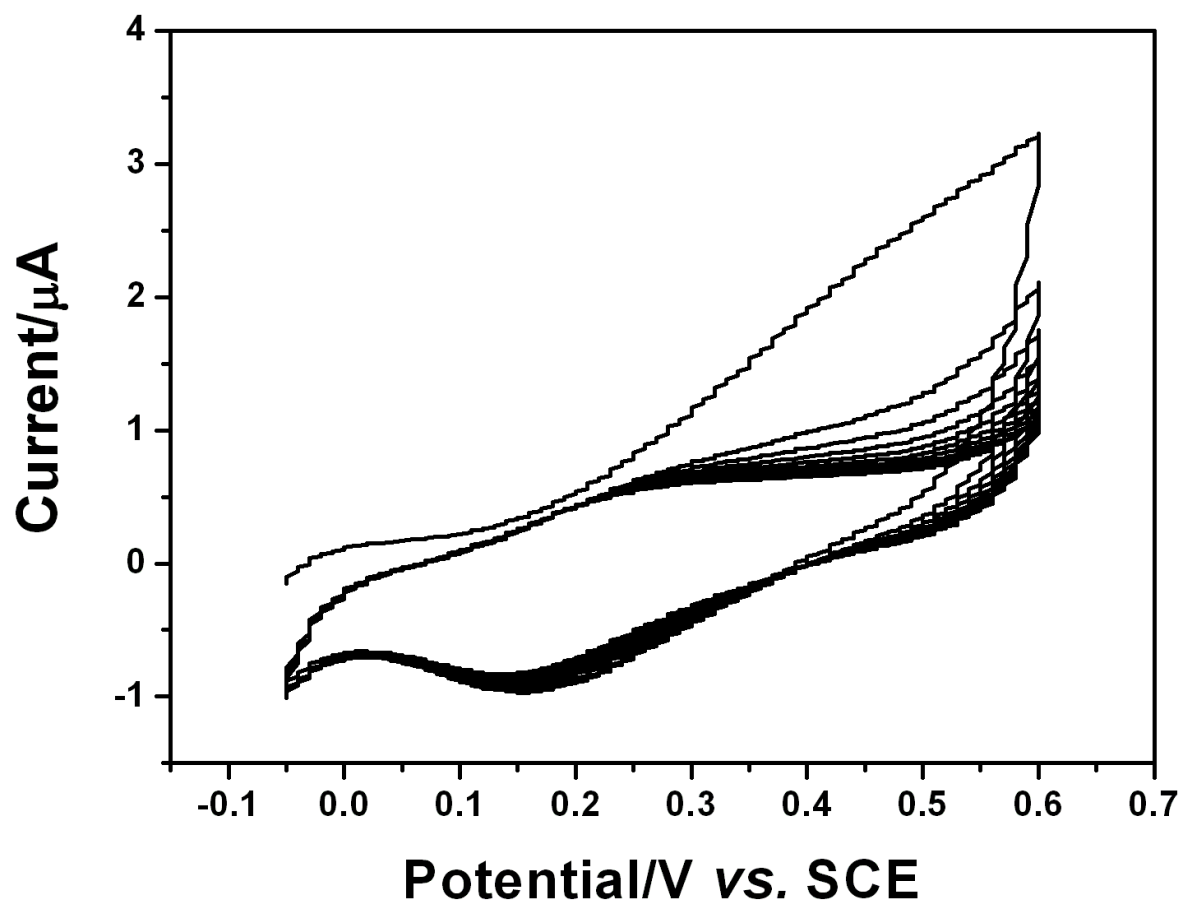


Figure 3

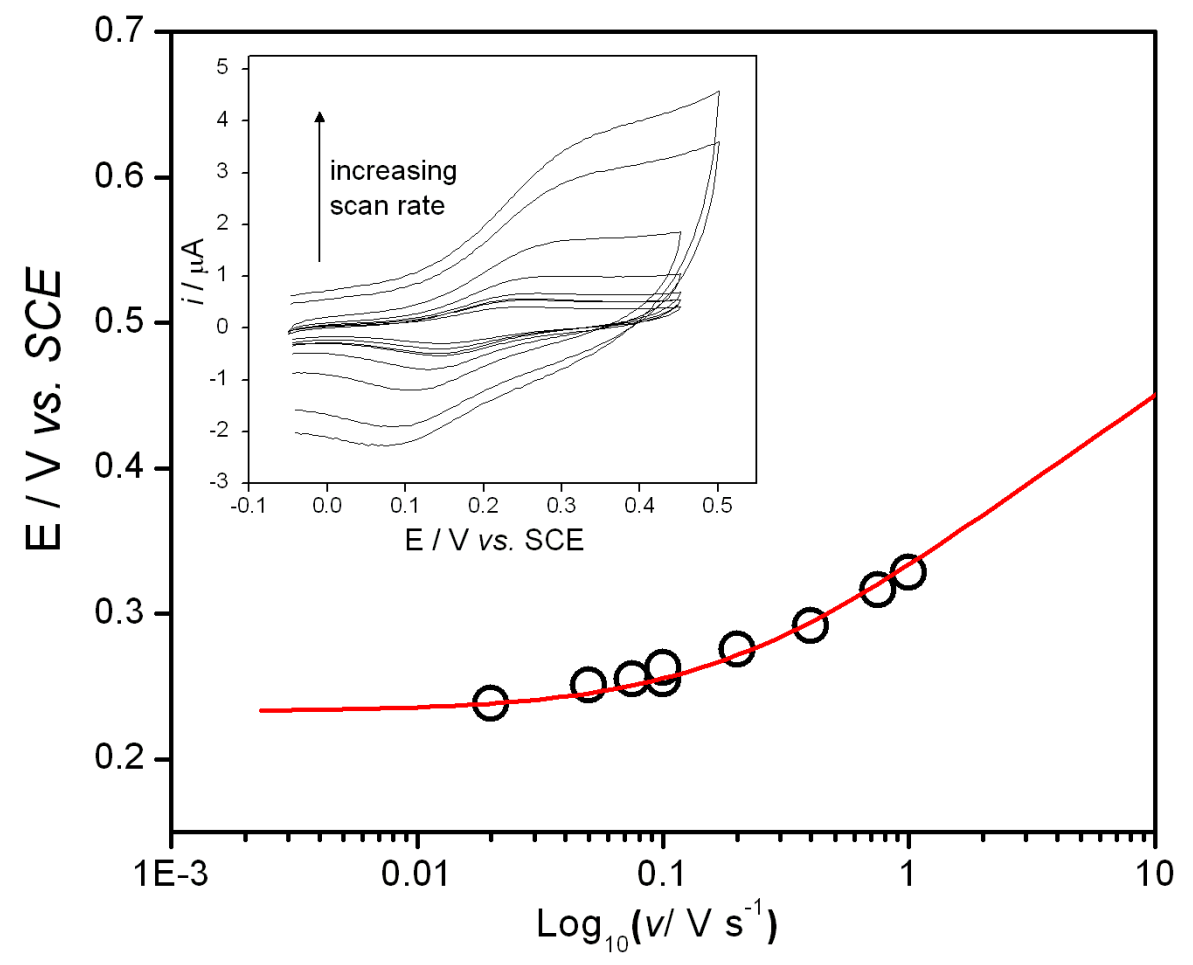


Figure 4

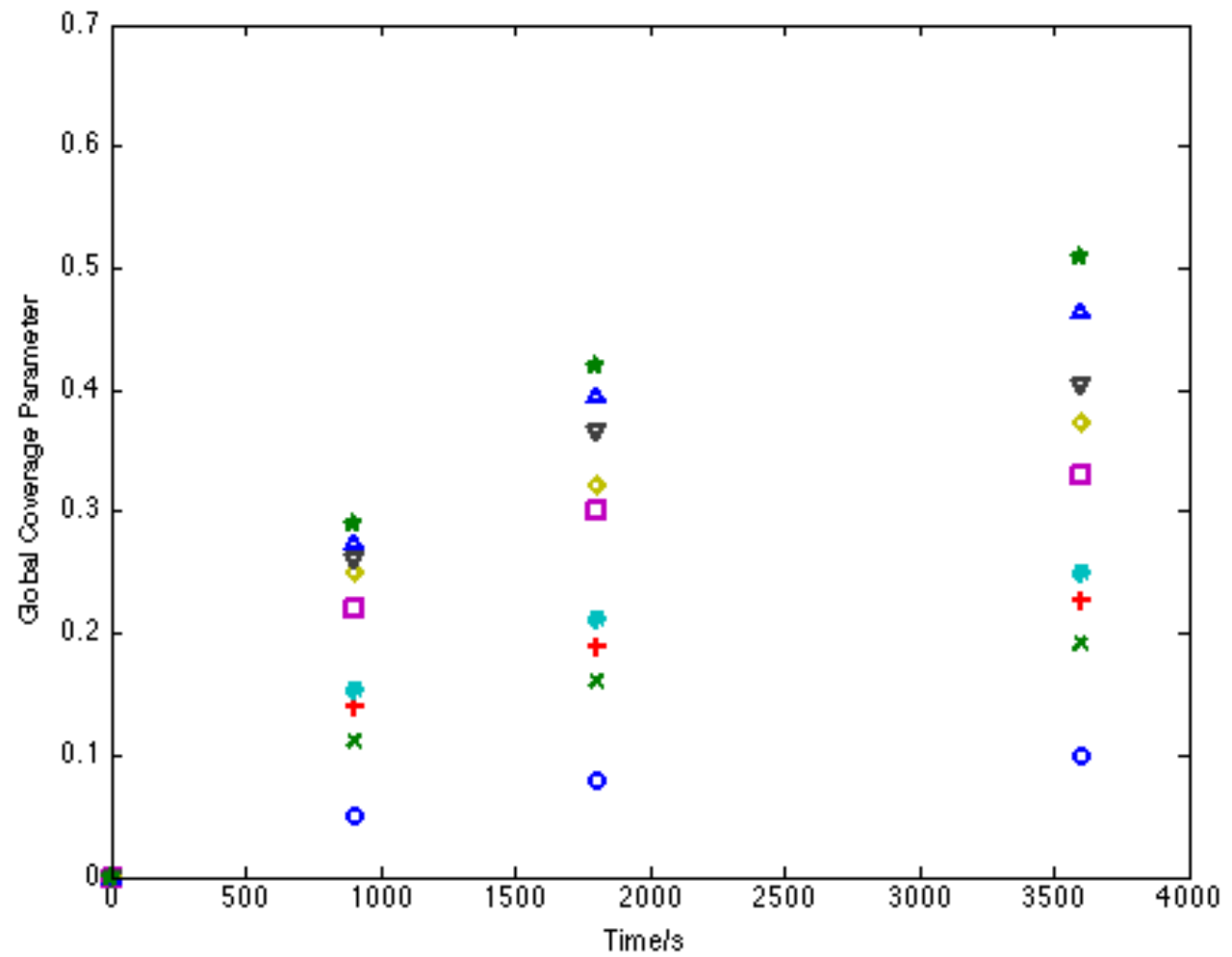


Figure 5

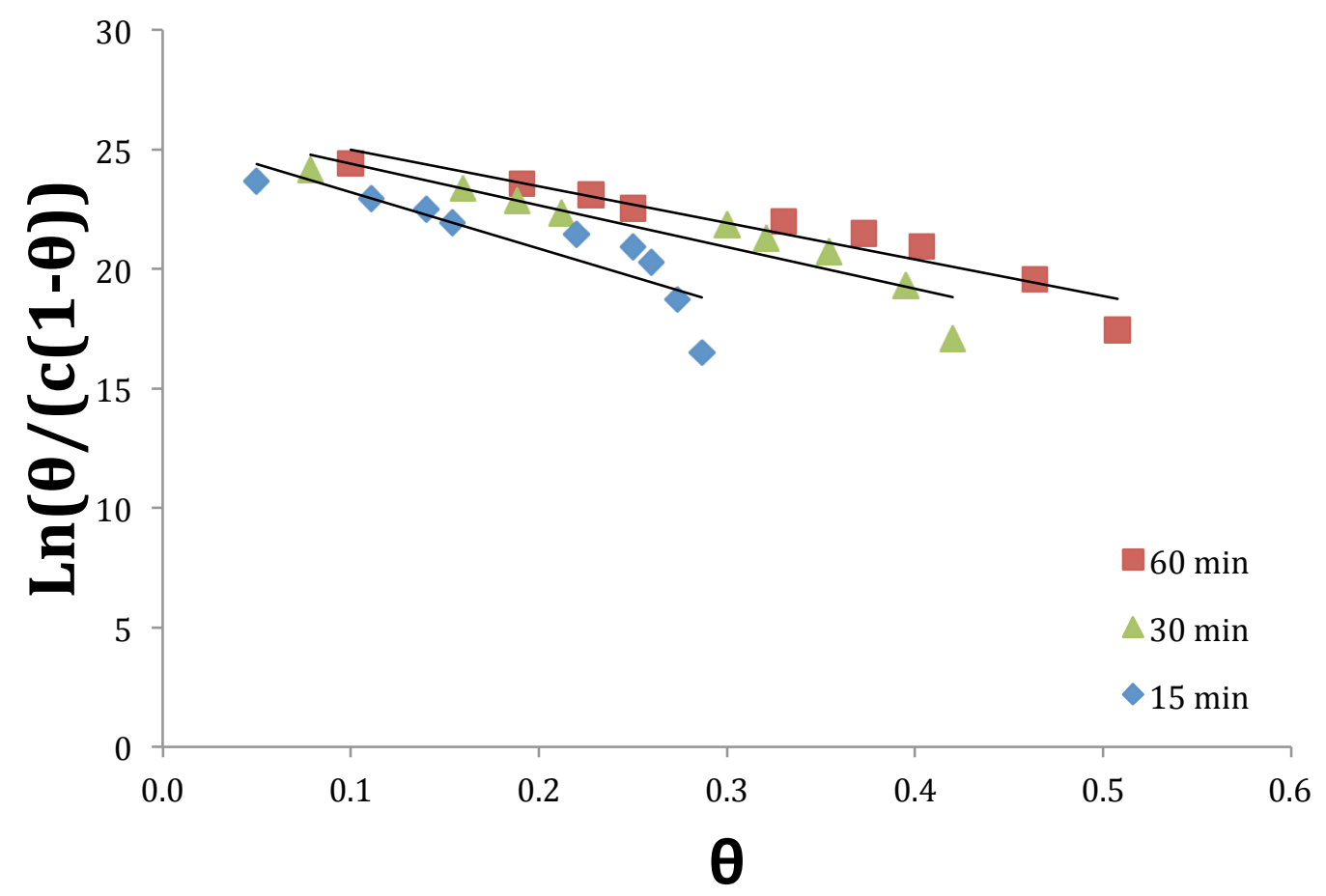


Figure 6

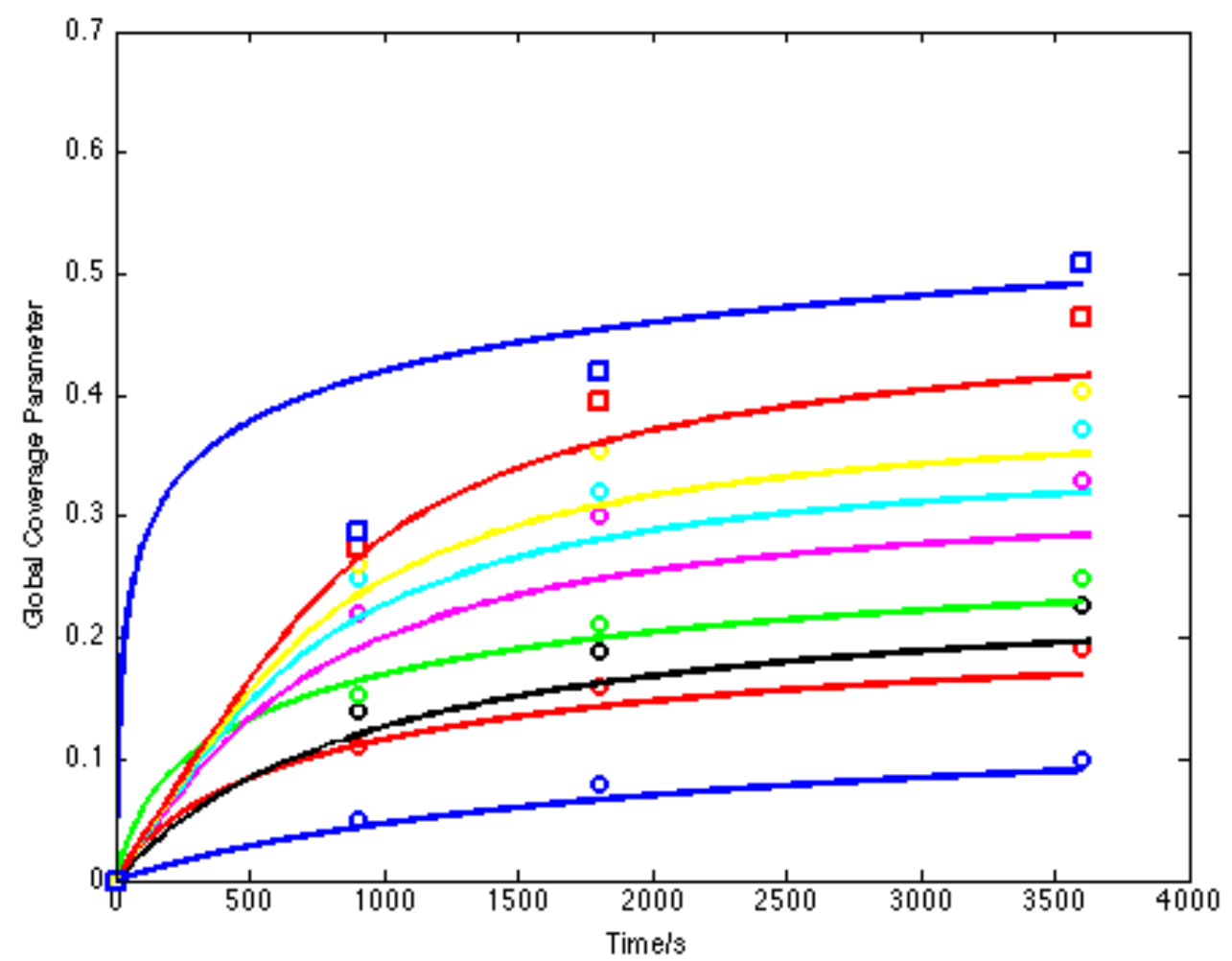




\section{Figure 7}

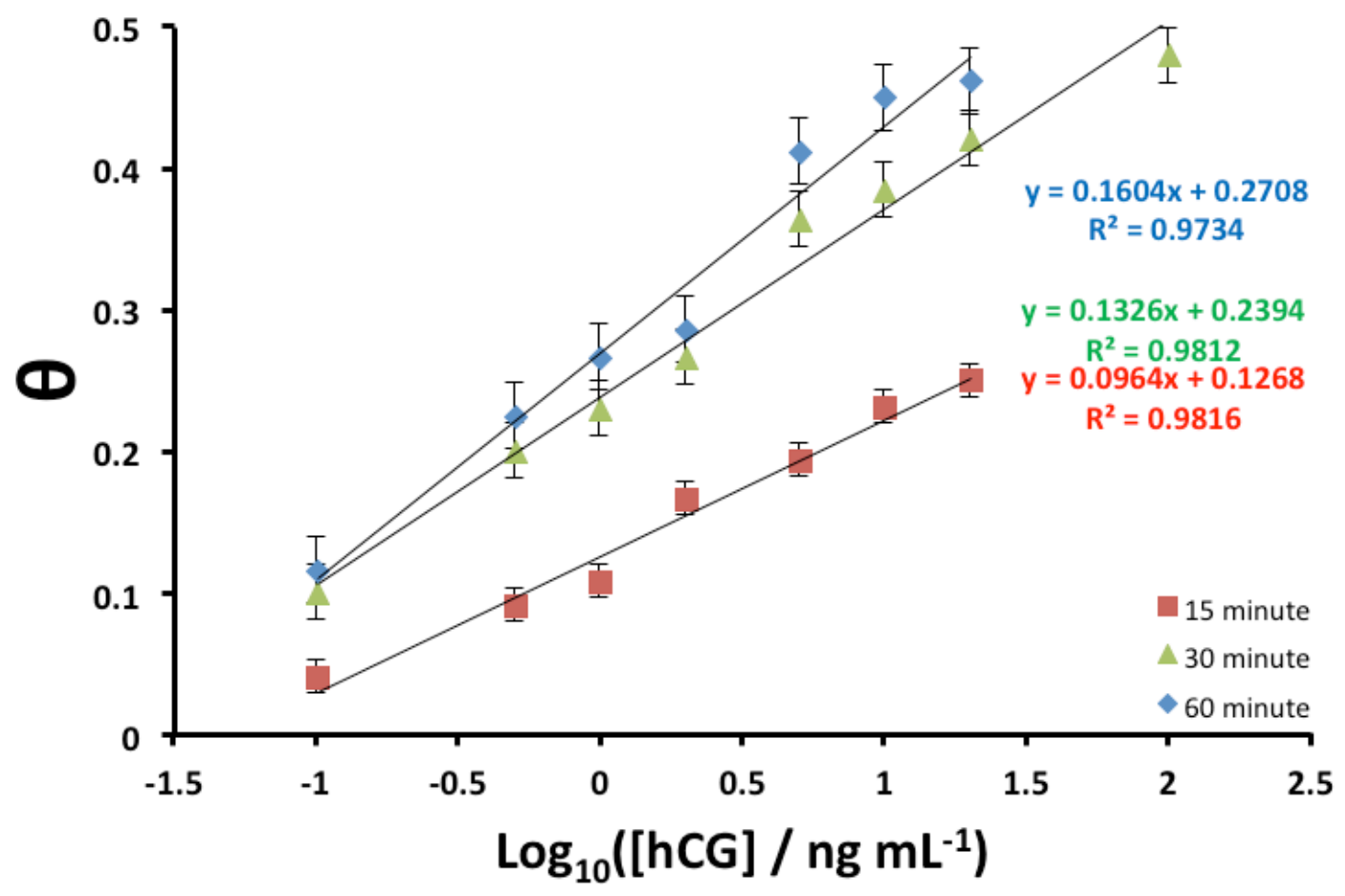

